\title{
Characteristics of isolated multiresistant bacteria according germ, location and "zero resistance" criteria
}

\author{
S Carvalho Brugger*, R Gavilan Rabell, M Miralbes Torner, G Jimenez Jimenez, S Iglesias Moles, J Trujillano Cabello, \\ M Vallverdú Vidal, B Balsera Garrido, F Barcenilla Gaite, M Palomar Martínez
}

From ESICM LIVES 2015

Berlin, Germany. 3-7 October 2015

\section{Introduction}

Multiresistant bacteria (MRB) development is a growing phenomenon. In 2013, the "Zero Resistance" (RZ) program was launched in Spain, to help prevent the emergence of multiresistant bacteria (MRB) in critically ill patients. One of its recommendations is to complete a checklist upon patient admission in Intensive Care Unit (ICU) to identify those patients at high risk for colonization or infection by MRB*.

\section{Objectives}

To search the relation between most common MRB and specific risk factors for colonization and infection, and the location of the samples where they are identified.

\section{Methods}

A prospective study from March $17^{\text {th }}, 2014$ to January $31^{\text {rst }}, 2015$. All patients admitted to a polyvalent ICU of a general hospital were submitted to the checklist proposed, with the application of contact precaution (CP) measures in patients at risk for colonization or infection by MRB. Bacteriologic swabs (nasal, pharyngeal, axillary and rectal) were routinely performed to all patients admitted, besides diagnostic cultures when needed. Furthermore, we analysed other pathological variables and comorbidities. The difference between groups of MRB was made by Chisquare test for qualitative variables and the Kruskal-Walls test for the continuous ones. Statistical significance was set at $\mathrm{p}<.05$.

\section{Results}

In 78 patients were identified one or more MRB (in total $91 \mathrm{MRB})$. They were classified in 6 groups. 29 patients (37,2\%) were MRSA carriers, $22(28,2 \%)$ E coli ESBLs, 10 (21,8\%) Klebsiella spp ESBLs, 10 (12,8\%) P aeruginosa,
8 (10,2\%) Acinetobacter spp and $12(15,4 \%)$ others MRB carriers (including 1 case of carbapenemase). Nasal swabs detected $26(33,3 \%)$ of MRB carriers [22 $(75,9 \%)$ of all MRSA], pharyngeal swabs $26(35,9 \%)$ [17 (43,6\%) of MRSA], axillary swabs $13(16,7 \%)$ [7 (24,1\%) of MRSA, $3(37,5 \%)$ of Acinetobacter spp], and rectal swabs 30 $(38,5 \%)[27(87,1 \%)$ of ESBLs, 3 (37,5\%) of Acinetobacterspp]. Diagnostic cultures (blood, urine, bronchoaspirate, surgical wound and others) detected MRB in less than 30\% of the cases. The checklist did not detected neither colonization nor infection by MRB in $29(37,2 \%)$ patients $(44,8 \%$ MRSA, 50\% Acinetobacter spp, 38,7\% of ESBLs). We did not find significant relation with other comorbidities.

\section{Conclusions}

In our environment, we detected a predominance of MRSA, higher than the Spanish media, followed by ESBLs. MRSA carriers were identified mostly by nasal swabs while the ESBL carriers were identified mainly by rectal swabs. We did not find significant relation between BMR and other comorbidities, possibly because of the sample size. The RZ checklist did not identify almost $40 \%$ of the MRB.

Published: 1 October 2015

\section{Reference}

1. Montero J, et al: (Scientific Expert Committee for the "Zero Resistance" Project). Combatting resistance in intensive care: the multimodal approach of the Spanish ICU "Zero Resistance" program. Critical Care 2015, 19:114.

doi:10.1186/2197-425X-3-S1-A134

Cite this article as: Carvalho Brugger et al: Characteristics of isolated multiresistant bacteria according germ, location and "zero resistance" criteria. Intensive Care Medicine Experimental 2015 3(Suppl 1):A134. 